\title{
The Exit Time and the Dividend Value Function for One-Dimensional Diffusion Processes
}

\author{
Peng Li, ${ }^{1}$ Chuancun Yin, ${ }^{1}$ and Ming Zhou ${ }^{2}$ \\ ${ }^{1}$ School of Mathematical Sciences, Qufu Normal University, Shandong 273165, China \\ ${ }^{2}$ China Institute for Actuarial Science, Central University of Finance and Economics, Beijing 100081, China
}

Correspondence should be addressed to Chuancun Yin; ccyin@mail.qfnu.edu.cn

Received 30 August 2013; Accepted 27 October 2013

Academic Editor: Yong Zhou

Copyright (c) 2013 Peng Li et al. This is an open access article distributed under the Creative Commons Attribution License, which permits unrestricted use, distribution, and reproduction in any medium, provided the original work is properly cited.

\begin{abstract}
We investigate the exit times from an interval for a general one-dimensional time-homogeneous diffusion process and their applications to the dividend problem in risk theory. Specifically, we first use Dynkin's formula to derive the ordinary differential equations satisfied by the Laplace transform of the exit times. Then, as some examples, we solve the closed-form expression of the Laplace transform of the exit times for several popular diffusions, which are commonly used in modelling of finance and insurance market. Most interestingly, as the applications of the exit times, we create the connect between the dividend value function and the Laplace transform of the exit times. Both the barrier and threshold dividend value function are clearly expressed in terms of the Laplace transform of the exit times.
\end{abstract}

\section{Introduction}

Diffusion processes have extensive applications in economics, finance, queueing, mathematical biology, and electric engineering. See, for example, [1-4] and the references therein. The main tool for studying various properties of diffusion is the result on exit times from an interval. Motivated by Yin et al. in [5], who considered the exit problems for jump processes with applications to dividend problems. In this paper, we consider the Laplace transforms of some random variables involving the exit time for the general onedimension diffusion processes with applications to dividend problems.

Let $U=\left\{U_{t}, t \geq 0\right\}$ be a one-dimensional time-homogeneous diffusion process, which is defined by the following stochastic differential equation:

$$
d U_{t}=\mu\left(U_{t}\right) d t+\sigma\left(U_{t}\right) d B_{t}, \quad U_{0}=u \in(a, b),
$$

where $B_{t}$ is a Brownian motion and $a<b$ are constants. It is well known that under certain conditions on the coefficients $\mu(u)$ and $\sigma(u)$, the SDE (1) has a unique strong solution for each starting point. The solution $U_{t}$ is a time-homogeneous strong Markov process with infinitesimal generator as follows:

$$
\mathscr{A} g(u)=\frac{1}{2} \sigma^{2}(u) g^{\prime \prime}(u)+\mu(u) g^{\prime}(u), \quad u \in(a, b),
$$

for any twice continuously differentiable function $g$.

Define

$$
\begin{gathered}
\tau_{a}^{-}=\inf \left\{t \geq 0: U_{t} \leq a\right\}, \\
\tau_{b}^{+}=\inf \left\{t \geq 0: U_{t} \geq b\right\}, \\
\tau_{a b}=\tau_{a}^{-} \wedge \tau_{b}^{+} .
\end{gathered}
$$

For $\delta>0$, we consider the following Laplace transforms:

$$
\begin{gathered}
\varphi_{1}(u)=E_{u}\left[e^{-\delta \tau_{a}^{-}}, \tau_{a}^{-}<\tau_{b}^{+}\right], \\
\varphi_{2}(u)=E_{u}\left[e^{-\delta \tau_{b}^{+}}, \tau_{b}^{+}<\tau_{a}^{-}\right], \\
\varphi(u)=E_{u}\left[e^{-\delta \tau_{a b}}\right]=\varphi_{1}(u)+\varphi_{2}(u) .
\end{gathered}
$$

We study the differential equations satisfied by the Laplace transforms and some applications of the popular dividend strategy in risk theory. 
The rest of the paper is organized as follows. Section 2 studies the Laplace transforms of exit times and considers some popular diffusions. Some applications in the calculation of dividend value functions for the barrier strategy and the threshold strategy are considered in Section 3.

\section{Laplace Transform}

In this section, we consider the Laplace transform of the exit time for the general diffusion process $\left\{U_{t} ; t \geq 0\right\}$ defined by (1).

Theorem 1. The function $\varphi_{1}(u)$ defined by (5) satisfies the following differential equation:

$$
\frac{1}{2} \sigma^{2}(u) \varphi_{1}^{\prime \prime}(u)+\mu(u) \varphi_{1}^{\prime}(u)=\delta \varphi_{1}(u), \quad u \in(a, b),
$$

with the boundary conditions $\varphi_{1}(a)=1, \varphi_{1}(b)=0$.

Proof. We assume that $f(u)$ is twice continuously differentiable and satisfies the following differential equation:

$$
\begin{gathered}
\mathscr{A} f(u)=\delta f(u), \quad u \in(a, b), \\
f(a)=1, \quad f(b)=0 .
\end{gathered}
$$

Applying Dynkin's formula to $h\left(t, U_{t}\right)=e^{-\delta t} f\left(U_{t}\right)$, we obtain

$$
\begin{aligned}
E_{u}\left[h\left(t, U_{t}\right)\right] & =h(0, u)+E_{u}\left(\int_{0}^{t}(\mathscr{A}-\delta) h\left(s, U_{s}\right) d s\right) \\
& =f(u)+E_{u}\left(\int_{0}^{t} e^{-\delta s}(\mathscr{A}-\delta) f\left(U_{s}\right) d s\right) .
\end{aligned}
$$

Since $\tau_{a b}<\infty$ is a stopping time, it follows from the optional sampling theorem that

$$
\begin{aligned}
E_{u} & {\left[e^{-\delta\left(\tau_{a b} \wedge t\right)} f\left(U_{\left(\tau_{a b} \wedge t\right)}\right)\right] } \\
& =f(u)+E_{u}\left(\int_{0}^{\left(\tau_{a b} \wedge t\right)} e^{-\delta s}(\mathscr{A}-\delta) f\left(U_{s}\right) d s\right),
\end{aligned}
$$

and letting $t \rightarrow \infty$, we get

$$
\begin{aligned}
E_{u} & {\left[e^{-\delta \tau_{a b}} f\left(U_{\tau_{a b}}\right)\right] } \\
& =f(u)+E_{u}\left(\int_{0}^{\tau_{a b}} e^{-\delta s}(\mathscr{A}-\delta) f\left(U_{s}\right) d s\right) .
\end{aligned}
$$

By the definitions of $\tau_{a b}$, we get

$$
\begin{aligned}
E_{u}\left[e^{-\delta \tau_{a b}} f\left(U_{\tau_{a b}}\right)\right]= & f(a) E_{u}\left[e^{-\delta \tau_{a}^{-}} ; \tau_{a}^{-}<\tau_{b}^{+}\right] \\
& +f(b) E_{u}\left[e^{-\delta \tau_{b}^{+}} ; \tau_{b}^{+}<\tau_{a}^{-}\right] .
\end{aligned}
$$

Substituting (9) and (10) into (13) and (14), we get

$$
f(u)=E_{u}\left[e^{-\delta \tau_{a}^{-}} ; \tau_{a}^{-}<\tau_{b}^{+}\right]=\varphi_{1}(u) .
$$

This completes the proof.
Theorem 2. The function $\varphi_{2}(u)=E_{u}\left[e^{-\delta \tau_{b}^{+}} ; \tau_{b}^{+}<\tau_{a}^{-}\right]$satisfies the following differential equation:

$$
\frac{1}{2} \sigma^{2}(u) \varphi_{2}^{\prime \prime}(u)+\mu(u) \varphi_{2}^{\prime}(u)=\delta \varphi_{2}(u), \quad u \in(a, b),
$$

with the boundary conditions $\varphi_{2}(a)=0, \varphi_{2}(b)=1$.

Proof. The proof of this theorem is similar to that of Theorem 1. We first assume that $f(u)$ is twice continuously differentiable and satisfies the following differential equation:

$$
\begin{gathered}
\mathscr{A} f(u)=\delta f(u), \quad u \in(a, b), \\
f(a)=0, \quad f(b)=1 .
\end{gathered}
$$

Applying Dynkin's formula to $e^{-\delta t} f\left(U_{t}\right)$, and after the same discussion as of Theorem 1, we also can obtain (13) and (14). Substituting (17) into (13) and (14), we get

$$
f(u)=E_{u}\left[e^{-\delta \tau_{b}^{+}} ; \tau_{b}^{+}<\tau_{a}^{-}\right]=\varphi_{2}(u) .
$$

This completes the proof.

According to the definition of (7), we can lead to the following theorem from Theorems 1 and 2.

Theorem 3. The function $\varphi(u)=E_{u}\left[e^{-\delta \tau_{a b}}\right]$ satisfies the following differential equation:

$$
\frac{1}{2} \sigma^{2}(u) \varphi^{\prime \prime}(u)+\mu(u) \varphi^{\prime}(u)=\delta \varphi(u), \quad u \in(a, b),
$$

with the boundary conditions $\varphi(a)=1, \varphi(b)=1$.

Now, we consider some examples.

Example 4. The Bessel process: $d U_{t}=\left((d-1) / 2 U_{t}\right) d t+d B_{t}$, where $d>1$ is a real number. We assume that $b>a>0$ in this process.

First, we consider the following differential equation:

$$
\frac{1}{2} f^{\prime \prime}(u)+\frac{d-1}{2 u} f^{\prime}(u)=\delta f(u) .
$$

It is well known that the increasing and decreasing solutions are, respectively, as follows:

$$
f_{+}(u)=u^{-v} I_{v}(\sqrt{2 \delta} u), \quad f_{-}(u)=u^{-v} K_{v}(\sqrt{2 \delta} u),
$$

where $v=(d-2) / 2$, and $I_{v}(\cdot)$ and $K_{v}(\cdot)$ are the usual modified Bessel functions.

Then, from Theorem 1 , we can give $\varphi_{1}(u)$ as follows:

$$
\begin{gathered}
\varphi_{1}(u)=C_{1} f_{+}(u)+C_{2} f_{-}(u), \\
\varphi_{1}(a)=1, \quad \varphi_{1}(b)=0,
\end{gathered}
$$


where the constants $C_{1}$ and $C_{2}$ are to be determined. From the boundary conditions (22), we can obtain the expression of the constants $C_{1}$ and $C_{2}$ as follows:

$$
\begin{aligned}
& C_{1}=\frac{a^{v} K_{v}(\sqrt{2 \delta} b)}{I_{v}(\sqrt{2 \delta} a) K_{v}(\sqrt{2 \delta} b)-I_{v}(\sqrt{2 \delta} b) K_{v}(\sqrt{2 \delta} a)}, \\
& C_{2}=\frac{a^{v} I_{v}(\sqrt{2 \delta} b)}{I_{v}(\sqrt{2 \delta} b) K_{v}(\sqrt{2 \delta} a)-I_{v}(\sqrt{2 \delta} a) K_{v}(\sqrt{2 \delta} b)} .
\end{aligned}
$$

So, we get

$\varphi_{1}(u)$

$$
=\left(\frac{a}{u}\right)^{v} \frac{K_{v}(\sqrt{2 \delta} b) I_{v}(\sqrt{2 \delta} u)-I_{v}(\sqrt{2 \delta} b) K_{v}(\sqrt{2 \delta} u)}{I_{v}(\sqrt{2 \delta} a) K_{v}(\sqrt{2 \delta} b)-I_{v}(\sqrt{2 \delta} b) K_{v}(\sqrt{2 \delta} a)} .
$$

According to Theorem 2 and (21), we can give $\varphi_{2}(u)$ as follows:

$$
\begin{gathered}
\varphi_{2}(u)=C_{3} f_{+}(u)+C_{4} f_{-}(u), \\
\varphi_{2}(a)=0, \quad \varphi_{2}(b)=1,
\end{gathered}
$$

where the constants $C_{3}$ and $C_{4}$ are to be determined. From the boundary conditions (25), we can determine the constants and obtain the expression of $\varphi_{2}(u)$ as follows:

$\varphi_{2}(u)$

$$
=\left(\frac{b}{u}\right)^{v} \frac{K_{v}(\sqrt{2 \delta} a) I_{v}(\sqrt{2 \delta} u)-I_{v}(\sqrt{2 \delta} a) K_{v}(\sqrt{2 \delta} u)}{I_{v}(\sqrt{2 \delta} b) K_{v}(\sqrt{2 \delta} a)-I_{v}(\sqrt{2 \delta} a) K_{v}(\sqrt{2 \delta} b)} .
$$

According to Theorem 3, the expression of $\varphi(u)$ can be obtained from solving the following differential equation:

$$
\begin{gathered}
\frac{1}{2} \varphi^{\prime \prime}(u)+\frac{d-1}{2 u} \varphi^{\prime}(u)=\delta \varphi(u), \\
\varphi(a)=1, \quad \varphi(b)=1 .
\end{gathered}
$$

Furthermore, from the definition of $\varphi(u)=\varphi_{1}(u)+\varphi_{2}(u)$, we also can get the expression of $\varphi(u)$. The two methods can lead to the same results as follows:

$$
\begin{aligned}
\varphi(u)= & \left(\frac{1}{u}\right)^{v} \\
& \times\left(\left[a^{v} K_{v}(\sqrt{2 \delta} b)-b^{v} K_{v}(\sqrt{2 \delta} a)\right] I_{v}(\sqrt{2 \delta} u)\right. \\
& \left.\quad+\left[b^{v} I_{v}(\sqrt{2 \delta} a)-a^{v} I_{v}(\sqrt{2 \delta} b)\right] K_{v}(\sqrt{2 \delta} u)\right) \\
& \times\left(I_{v}(\sqrt{2 \delta} a) K_{v}(\sqrt{2 \delta} b)-I_{v}(\sqrt{2 \delta} b) K_{v}(\sqrt{2 \delta} a)\right)^{-1} .
\end{aligned}
$$

Example 5 (the square root process (see [6])).

$$
d U_{t}=v\left(k-U_{t}\right) d t+\sigma \sqrt{U_{t}} d B_{t}, \quad v, \sigma>0, k \in[a, b] .
$$

We assume that $b>a>0$ and consider the following differential equation:

$$
\frac{1}{2} \sigma^{2} u^{2} f^{\prime \prime}(u)+(v k-v u) f^{\prime}(u)=\delta f(u), \quad \delta>0 .
$$

We assume that $\left(2 \nu / \sigma^{2}\right) k$ is not an integer, the two linear independent solutions are

$$
\begin{aligned}
& f_{+}(u)=M\left(\frac{\delta}{v}, \frac{2 v}{\sigma^{2}} k, \frac{2 v}{\sigma^{2}} u\right), \\
& f_{-}(u)=U\left(\frac{\delta}{v}, \frac{2 v}{\sigma^{2}} k, \frac{2 v}{\sigma^{2}} u\right),
\end{aligned}
$$

where $M$ and $U$ are the confluent hypergeometric functions of the first and second kinds, respectively. Then, as the way at used in Example 4, and from Theorems 1 and 2, we get that the expressions of $\varphi_{1}(u)$ and $\varphi_{2}(u)$ are as follows:

$$
\begin{gathered}
\varphi_{1}(u)=\frac{g(b, u)-g(u, b)}{g(b, a)-g(a, b)}, \\
\varphi_{2}(u)=\frac{g(a, u)-g(u, a)}{g(a, b)-g(b, a)},
\end{gathered}
$$

where

$$
g(x, y)=U\left(\frac{\delta}{v}, \frac{2 v}{\sigma^{2}} k, \frac{2 v}{\sigma^{2}} x\right) M\left(\frac{\delta}{v}, \frac{2 v}{\sigma^{2}} k, \frac{2 v}{\sigma^{2}} y\right) .
$$

So, we can get

$$
\varphi(u)=\frac{g(a, u)-g(b, u)+g(u, b)-g(u, a)}{g(a, b)-g(b, a)} .
$$

Example 6 (the Ornstein-Uhlenbeck process (see [7])).

$$
d U_{t}=v\left(k-U_{t}\right) d t+\sigma d B_{t}, \quad v, \sigma>0, k \in[a, b] .
$$

The Ornstein-Uhlenbeck process above is the only process that is simultaneously Gaussian, Markov, and stationary, and has been discussed extensively, see, for example $[2-4,8]$.

We consider the following differential equation:

$$
\frac{1}{2} \sigma^{2} f^{\prime \prime}(u)+v(k-u) f^{\prime}(u)=\delta f(u) .
$$

In the case of $k=0, \sigma=1$, the two independent solutions to

$$
\frac{1}{2} f^{\prime \prime}(u)-v u f^{\prime}(u)=\delta f(u)
$$

are

$$
\begin{gathered}
f_{+}(u)=H_{-\delta / v}(-\sqrt{v} u)=2^{-\delta / 2 v} e^{(1 / 2) v u^{2}} D_{-\delta / v}(-\sqrt{2 v} u), \\
f_{-}(u)=H_{-\delta / v}(\sqrt{v} u)=2^{-\delta / 2 v} e^{(1 / 2) v u^{2}} D_{-\delta / v}(\sqrt{2 v} u),
\end{gathered}
$$


where $H_{v}(\cdot)$ and $D_{v}(\cdot)$ are, respectively, the Hermite and parabolic functions. We obtain the expressions of $\varphi_{1}(u)$ and $\varphi_{2}(u)$ as follows:

$$
\begin{aligned}
& \varphi_{1}(u)=e^{(1 / 2) v\left(u^{2}-a^{2}\right)} \frac{h(u, b)-h(b, u)}{h(a, b)-h(b, a)}, \\
& \varphi_{2}(u)=e^{(1 / 2) v\left(u^{2}-b^{2}\right)} \frac{h(a, u)-h(u, a)}{h(a, b)-h(b, a)},
\end{aligned}
$$

where

$$
h(x, y)=D_{-\delta / v}(-\sqrt{2 v} x) D_{-\delta / v}(\sqrt{2 v} y) .
$$

By the definition of $\varphi(u)$, we get

$$
\begin{aligned}
\varphi(u)=\left(e^{(1 / 2) v\left(u^{2}-a^{2}\right)}(h(u, b)-h(b, u))\right. \\
\left.+e^{(1 / 2) v\left(u^{2}-b^{2}\right)}(h(a, u)-h(u, a))\right) \\
\times(h(a, b)-h(b, a))^{-1} .
\end{aligned}
$$

For the general $k$ and $\sigma$, the two independent solutions of (36) are, respectively, as follows:

$$
\begin{aligned}
f_{+}(u) & =H_{-\delta / v}\left(-\frac{\sqrt{v}}{\sigma}(u-k)\right) \\
& =2^{-\delta / 2 v} e^{(1 / 2)\left(v / \sigma^{2}\right)(u-k)^{2}} D_{-\delta / v}\left(-\frac{\sqrt{2 v}}{\sigma}(u-k)\right), \\
f_{-}(u) & =H_{-\delta / v}\left(\frac{\sqrt{v}}{\sigma}(u-k)\right) \\
& =2^{-\delta / 2 v} e^{(1 / 2)\left(v / \sigma^{2}\right)(u-k)^{2}} D_{-\delta / v}\left(\frac{\sqrt{2 v}}{\sigma}(u-k)\right) .
\end{aligned}
$$

Then, we obtain the expressions of $\varphi_{1}(u)$ and $\varphi_{2}(u)$ as follows:

$$
\begin{aligned}
& \varphi_{1}(u)=e^{(1 / 2)\left(v / \sigma^{2}\right)\left[(u-k)^{2}-(a-k)^{2}\right]} \frac{h_{0}(u, b)-h_{0}(b, u)}{h_{0}(a, b)-h_{0}(b, a)}, \\
& \varphi_{2}(u)=e^{(1 / 2)\left(v / \sigma^{2}\right)\left[(u-k)^{2}-(b-k)^{2}\right]} \frac{h_{0}(a, u)-h_{0}(u, a)}{h_{0}(a, b)-h_{0}(b, a)},
\end{aligned}
$$

where

$$
h_{0}(x, y)=D_{-\delta / v}\left(-\frac{\sqrt{2 v}}{\sigma}(x-k)\right) D_{-\delta / v}\left(\frac{\sqrt{2 v}}{\sigma}(y-k)\right) .
$$

Finally, we get

$$
\begin{aligned}
\varphi(u)= & e^{(1 / 2)\left(v / \sigma^{2}\right)\left[(u-k)^{2}-(a-k)^{2}\right]} \frac{h_{0}(u, b)-h_{0}(b, u)}{h_{0}(a, b)-h_{0}(b, a)} \\
& +e^{(1 / 2)\left(v / \sigma^{2}\right)\left[(u-k)^{2}-(b-k)^{2}\right]} \frac{h_{0}(a, u)-h_{0}(u, a)}{h_{0}(a, b)-h_{0}(b, a)} .
\end{aligned}
$$

Example 7 (the Gompertz Brownian motion process (see [9])).

$d U_{t}=v U_{t}\left(\ln k-\ln U_{t}\right) d t+\sigma U_{t} d B_{t}, \quad v, \sigma>0, k \in[a, b]$.

We assume that $b>a>0$.

Now, consider the differential equation

$$
\frac{1}{2} \sigma^{2} u^{2} f^{\prime \prime}(u)+v u\left(\ln k-\ln U_{t}\right) f^{\prime}(u)=\delta f(u), \quad \delta>0 .
$$

It is well known that the increasing and decreasing solutions are, respectively, as follows:

$$
\begin{aligned}
& f_{+}(u)=M\left(\frac{\delta}{2 v}, \frac{1}{2}, \frac{v}{\sigma^{2}}\left(\ln \frac{u}{k}+\frac{\sigma^{2}}{2 v}\right)^{2}\right), \\
& f_{-}(u)=U\left(\frac{\delta}{2 v}, \frac{1}{2}, \frac{v}{\sigma^{2}}\left(\ln \frac{u}{k}+\frac{\sigma^{2}}{2 v}\right)^{2}\right),
\end{aligned}
$$

where $M$ and $U$, as in Example 5, are the first and second Kummer's function, respectively. From the boundary conditions $\varphi_{1}(a)=1$ and $\varphi_{1}(b)=0$, we get

$$
\varphi_{1}(u)=\frac{g_{0}(b, u)-g_{0}(u, b)}{g_{0}(b, a)-g_{0}(a, b)},
$$

where

$$
\begin{aligned}
g_{0}(x, y)= & U\left(\frac{\delta}{2 v}, \frac{1}{2}, \frac{v}{\sigma^{2}}\left(\ln \frac{x}{k}+\frac{\sigma^{2}}{2 v}\right)^{2}\right) \\
& \times M\left(\frac{\delta}{2 v}, \frac{1}{2}, \frac{v}{\sigma^{2}}\left(\ln \frac{y}{k}+\frac{\sigma^{2}}{2 v}\right)^{2}\right) .
\end{aligned}
$$

From the boundary conditions $\varphi_{2}(a)=0$ and $\varphi_{2}(b)=1$, we get

$$
\varphi_{2}(u)=\frac{g_{0}(u, a)-g_{0}(a, u)}{g_{0}(b, a)-g_{0}(a, b)} .
$$

Then, we obtain

$$
\varphi(u)=\frac{g_{0}(b, u)-g_{0}(a, u)+g_{0}(u, a)-g_{0}(u, b)}{g_{0}(b, a)-g_{0}(a, b)} .
$$

\section{Applications to Dividend Value Function}

3.1. Barrier Strategy. In this subsection, we consider the barrier strategy for dividend payments which are discussed in various model, see, for example, [10-13]. More specifically, we assume that the company pays dividends according to the following strategy governed by parameter $b>0$. Whenever the surplus is above the level $b$, the excess will be paid as dividends, and when the surplus is below $b$ nothing is paid out. We denote the aggregate dividends paid in the time interval $[0, t]$ by $D_{r}(t)$, the modified risk process by 
$U_{r}(t)=U_{t}-D_{r}(t)$, the ruin time by $T_{r}=\inf \{t \geq 0$ : $\left.U_{r}(t)=0\right\}$, and the present value of all dividends until ruin $T_{r}$ by $D_{r}=\int_{0}^{T_{r}} e^{-\delta t} d D_{r}(t)$, here, $\delta>0$ is the discount factor, and the expectation of $D_{r}$ by

$$
V_{r}(u, b)=E_{u}\left[D_{r}\right] .
$$

Now, we want to derive the dividend value function by the Laplace transform of exit time. We denote

$$
\begin{gathered}
\tau_{0}^{-}=\inf \left\{t \geq 0: U_{t} \leq 0\right\}, \\
\tau_{0 b}=\tau_{0}^{-} \wedge \tau_{b}^{+}, \\
\psi_{2}(u)=E\left[e^{-\delta \tau_{b}^{+}} ; \tau_{b}^{+}<\tau_{0}^{-}\right],
\end{gathered}
$$

where $\tau_{b}^{+}$is defined by (3). Let $a=0$ in the function $\varphi_{2}(u)$ be defined by (6), we get the definition of $\psi_{2}(u)$. So, we get the following lemma from Theorem 2.

Lemma 8. The function $\psi_{2}(u)$ defined by (56) satisfies the following differential equation:

$$
\frac{1}{2} \sigma^{2}(u) \psi_{2}^{\prime \prime}(u)+\mu(u) \psi_{2}^{\prime}(u)=\delta \psi_{2}(u), \quad u \in(0, b),
$$

with the boundary conditions $\psi_{2}(0)=0, \psi_{2}(b)=1$.

Then, we have the following theorem.

Theorem 9. For $0 \leq u \leq b$, one has

$$
V_{r}(u, b)=\frac{\psi_{2}(u)}{\psi_{2}^{\prime}(b)},
$$

where $\psi_{2}(u)$ is defined by (56).

Proof. The one-dimensional diffusion model defined by (1) is a time-homogeneous strong Markov process. Then, when $0<u<b$, we have

$$
\begin{aligned}
V_{r}(u, b) & =E_{u}\left(\int_{0}^{T_{r}} e^{-\delta t} d D_{r}(t)\right) \\
& =E_{u}\left(\int_{0}^{\tau_{0 b}} e^{-\delta t} d D_{r}(t)+\int_{\tau_{0 b}}^{T_{r}} e^{-\delta t} d D_{r}(t)\right) \\
& =E_{u}\left(\int_{\tau_{0 b}}^{T_{r}} e^{-\delta t} d D_{r}(t)\right) \\
& =E_{u}\left[e^{-\delta \tau_{0 b}}\left(\int_{0}^{T_{r}} e^{-\delta t} d D_{r}(t) \circ \theta_{\tau_{0 b}}\right)\right] \\
& =E_{u}\left[e^{-\delta \tau_{0 b}} E_{U_{\tau_{0 b}}}\left(\int_{0}^{T_{r}} e^{-\delta t} d D_{r}(t)\right)\right] \\
& =E_{u}\left[e^{-\delta \tau_{0 b}} V_{r}\left(U_{\tau_{0 b}}, b\right)\right],
\end{aligned}
$$

where $\theta \tau_{0 b}$ is the shift operator. By the definition of $\tau_{0 b}$, we get

$$
\begin{aligned}
E_{u}\left[e^{\tau_{0 b}} V_{r}\left(U_{\tau_{0 b}}, b\right)\right]= & V_{r}(0, b) E_{u}\left[e^{-\delta \tau_{0}^{-}} ; \tau_{0}^{-}<\tau_{b}^{+}\right] \\
& +V_{r}(b, b) E_{u}\left[e^{-\delta \tau_{b}^{+}} ; \tau_{b}^{+}<\tau_{0}^{-}\right] \\
= & V_{r}(b, b) \psi_{2}(u) .
\end{aligned}
$$

From (59) and (60), we obtain

$$
V_{r}(u, b)=V_{r}(b, b) \psi_{2}(u),
$$

where $\psi_{2}(u)$ can be determined from Lemma 8. From [11], for the barrier strategy, we have the following boundary condition:

$$
\left.\frac{\partial V(u, b)}{\partial u}\right|_{u=b}=1
$$

Then, we have

$$
V_{r}(b, b)=\frac{1}{\psi_{2}^{\prime}(b)} .
$$

So, we get the result. This completes the proof.

Now, we consider the examples discussed in Section 2.

Example 10. The Bessel process discussed in Example 4. Reference [14] gives the following helpful formulas:

$$
I_{0}(u) \approx 1,
$$

$$
\begin{gathered}
I_{v}(u) \approx \frac{(u / 2)^{2}}{\Gamma(1+v)} \quad(v \neq-1,-2, \ldots), \\
K_{0}(u) \approx-\ln u, \\
K_{v}(u) \approx \frac{\Gamma(v)}{2}\left(\frac{u}{2}\right)^{-v} \quad(\operatorname{Re}(v)>0) .
\end{gathered}
$$

Letting $a=0$ in Example 4, we get $\psi_{2}(u)$ from $\varphi_{2}(u)$ as follows:

$$
\psi_{2}(u)=\left(\frac{b}{u}\right)^{v} \frac{I_{v}(\sqrt{2 \delta} u)}{I_{v}(\sqrt{2 \delta} b)} .
$$

Using the following formula:

$$
I_{v}^{\prime}(u)=\frac{v}{u} I_{v}(u)+I_{v+1}(u),
$$

we have

$$
\psi_{2}^{\prime}(b)=\left(\frac{b}{u}\right)^{v} \frac{\sqrt{2 \delta I_{v+1}(\sqrt{2 \delta} b)}}{I_{v}(\sqrt{2 \delta} b)} .
$$

Then, from Theorem 9 , and substituting $\psi_{2}(u)$ and $\psi_{2}^{\prime}(b)$ into (58), we obtain

$$
V_{r}(u, b)=\frac{I_{v}(\sqrt{2 \delta} u)}{\sqrt{2 \delta I_{v+1}(\sqrt{2 \delta} b)}} .
$$


Example 11. We consider the square root process discussed in Example 5. Let $a=0$ in Example 5, according to $M(x, y, 0)=$ 1 , we obtain

$$
\begin{gathered}
\psi_{2}(u)=\frac{1}{C}\left[U\left(\frac{\delta}{v}, \frac{2 v}{\sigma^{2}} k, 0\right) M\left(\frac{\delta}{v}, \frac{2 v}{\sigma^{2}} k, \frac{2 v}{\sigma^{2}} u\right)\right. \\
\left.-U\left(\frac{\delta}{v}, \frac{2 v}{\sigma^{2}} k, \frac{2 v}{\sigma^{2}} u\right)\right],
\end{gathered}
$$

where

$$
C=U\left(\frac{\delta}{v}, \frac{2 v}{\sigma^{2}} k, 0\right) M\left(\frac{\delta}{v}, \frac{2 v}{\sigma^{2}} k, \frac{2 v}{\sigma^{2}} b\right)-U\left(\frac{\delta}{v}, \frac{2 v}{\sigma^{2}} k, \frac{2 v}{\sigma^{2}} b\right) .
$$

Using the following helpful formulas (see [14]):

$$
\begin{aligned}
& \frac{\partial M\left(c_{1}, c_{2}, u\right)}{\partial u}=\frac{c_{1}}{c_{2}} M\left(c_{1}+1, c_{2}+1, u\right), \\
& \frac{\partial U\left(c_{1}, c_{2}, u\right)}{\partial u}=-c_{1} U\left(c_{1}+1, c_{2}+1, u\right),
\end{aligned}
$$

we get

$$
\begin{gathered}
\psi_{2}^{\prime}(b)=\frac{1}{C}\left[\frac{\delta}{v k} U\left(\frac{\delta}{v}, \frac{2 v}{\sigma^{2}} k, 0\right) M\left(\frac{\delta}{v}+1, \frac{2 v}{\sigma^{2}} k+1, \frac{2 v}{\sigma^{2}} b\right)\right. \\
\left.+\frac{2 \delta}{\sigma^{2}} U\left(\frac{\delta}{v}+1, \frac{2 v}{\sigma^{2}} k+1, \frac{2 v}{\sigma^{2}} b\right)\right] .
\end{gathered}
$$

Finally, we have

$$
\begin{aligned}
& V_{r}(u, b)=\left[U\left(\frac{\delta}{v}, \frac{2 v}{\sigma^{2}} k, 0\right) M\left(\frac{\delta}{v}, \frac{2 v}{\sigma^{2}} k, \frac{2 v}{\sigma^{2}} u\right)\right. \\
&\left.-U\left(\frac{\delta}{v}, \frac{2 v}{\sigma^{2}} k, \frac{2 v}{\sigma^{2}} u\right)\right] \\
& \times {\left[\frac{\delta}{v k} U\left(\frac{\delta}{v}, \frac{2 v}{\sigma^{2}} k, 0\right) M\left(\frac{\delta}{v}+1, \frac{2 v}{\sigma^{2}} k+1, \frac{2 v}{\sigma^{2}} b\right)\right.} \\
&\left.+\frac{2 \delta}{\sigma^{2}} U\left(\frac{\delta}{v}+1, \frac{2 v}{\sigma^{2}} k+1, \frac{2 v}{\sigma^{2}} b\right)\right]^{-1} .
\end{aligned}
$$

Example 12. We consider the Ornstein-Uhlenbeck process considered in Example 6, and let $a=0$ in Example 6. From [14], we have the following formulas:

$$
\begin{gathered}
D_{v}(0)=\frac{2^{v / 2} \sqrt{\pi}}{\Gamma((1 / 2)-(v / 2))}, \\
D_{v}^{\prime}(u)=-\frac{u}{2} D_{v}(u)+v D_{v-1}(u),
\end{gathered}
$$

where $\Gamma(x)$ is the gamma function.
In the case of $k=0, \sigma=1$, we have

$$
\begin{aligned}
& \psi_{2}(u)=e^{(v / 2)\left(u^{2}-b^{2}\right)} \frac{D_{-\delta / v}(\sqrt{2 v} u)-D_{-\delta / v}(-\sqrt{2 v} u)}{D_{-\delta / v}(\sqrt{2 v} b)-D_{-\delta / v}(-\sqrt{2 v} b)}, \\
& \psi_{2}^{\prime}(b)=\delta \sqrt{\frac{2}{v}} \frac{D_{(-\delta / v)-1}(\sqrt{2 v} b)+D_{(-\delta / v)-1}(-\sqrt{2 v} b)}{D_{-\delta / v}(-\sqrt{2 v} b)-D_{-\delta / v}(\sqrt{2 v} b)} .
\end{aligned}
$$

Then, we get

$$
\begin{aligned}
V_{r}(u, b)= & \frac{1}{\delta} \sqrt{\frac{v}{2}} e^{(v / 2)\left(u^{2}-b^{2}\right)} \\
& \times \frac{D_{-\delta / v}(-\sqrt{2 v} u)-D_{-\delta / v}(\sqrt{2 v} u)}{D_{(-\delta / v)-1}(\sqrt{2 v} b)+D_{(-\delta / v)-1}(-\sqrt{2 v} b)} .
\end{aligned}
$$

For the general $k, \sigma$, we have

$$
\begin{aligned}
\psi_{2}(u)= & \frac{1}{G} e^{\left(v / 2 \sigma^{2}\right)\left((u-k)^{2}-(b-k)^{2}\right)} \\
& \times\left[D_{-\delta / v}\left(\left(\frac{\sqrt{2 v}}{\sigma}\right) k\right) D_{-\delta / v}\left(\left(\frac{\sqrt{2 v}}{\sigma}\right)(u-k)\right)\right. \\
& -D_{-\delta / v}\left(-\left(\frac{\sqrt{2 v}}{\sigma}\right) k\right) \\
& \left.\times D_{-\delta / v}\left(\left(-\frac{\sqrt{2 v}}{\sigma}\right)(u-k)\right)\right]
\end{aligned}
$$

where

$$
\begin{aligned}
G= & D_{-\delta / v}\left(\left(\frac{\sqrt{2 v}}{\sigma}\right) k\right) D_{-\delta / v}\left(\left(\frac{\sqrt{2 v}}{\sigma}\right)(b-k)\right) \\
& -D_{-\delta / v}\left(-\left(\frac{\sqrt{2 v}}{\sigma}\right) k\right) D_{-\delta / v}\left(-\left(\frac{\sqrt{2 v}}{\sigma}\right)(u-k)\right) .
\end{aligned}
$$

We can get

$$
\begin{aligned}
\psi_{2}^{\prime}(b)= & -\frac{1}{G} \frac{\delta}{\sigma} \sqrt{\frac{2}{v}} \\
& \times\left[D_{-\delta / v}\left(\left(\frac{\sqrt{2 v}}{\sigma}\right) k\right) D_{(-\delta / v)-1}\left(\left(\frac{\sqrt{2 v}}{\sigma}\right)(b-k)\right)\right. \\
& +D_{-\delta / v}\left(-\left(\frac{\sqrt{2 v}}{\sigma}\right) k\right) \\
& \left.\times D_{(-\delta / v)-1}\left(-\left(\frac{\sqrt{2 v}}{\sigma}\right)(b-k)\right)\right]
\end{aligned}
$$


Finally, we obtain

$$
\begin{aligned}
& V_{r}(u, b)=\frac{1}{G} e^{\left(v / 2 \sigma^{2}\right)\left((u-k)^{2}-(b-k)^{2}\right)} \frac{\sigma}{\delta} \sqrt{\frac{v}{2}} \\
& \times\left[D_{-\delta / v}\left(-\left(\frac{\sqrt{2 v}}{\sigma}\right) k\right)\right. \\
& \times D_{-\delta / v}\left(-\left(\frac{\sqrt{2 v}}{\sigma}\right)(u-k)\right) \\
& -D_{-\delta / v}\left(\left(\frac{\sqrt{2 v}}{\sigma}\right) k\right) \\
& \left.\times D_{-\delta / v}\left(\left(\frac{\sqrt{2 v}}{\sigma}\right)(u-k)\right)\right] \\
& \times\left[D_{-\delta / v}\left(\left(\frac{\sqrt{2 v}}{\sigma}\right) k\right)\right. \\
& \times D_{(-\delta / v)-1}\left(\left(\frac{\sqrt{2 v}}{\sigma}\right)(b-k)\right) \\
& +D_{-\delta / v}\left(-\left(\frac{\sqrt{2 v}}{\sigma}\right) k\right) \\
& \left.\times D_{(-\delta / v)-1}\left(-\left(\frac{\sqrt{2 v}}{\sigma}\right)(b-k)\right)\right]^{-1} .
\end{aligned}
$$

Example 13. We consider the Gompertz Brownian motion process discussed in Example 7. In [15], the authors point out as $u \rightarrow+\infty$,

$$
\begin{gathered}
U\left(c_{1}, c_{2}, u\right)=u^{-c_{1}}\left[1+o\left(|u|^{-1}\right)\right], \\
M\left(c_{1}, c_{2}, u\right)=\frac{\Gamma\left(c_{2}\right)}{\Gamma\left(c_{1}\right)} e^{u} u^{c_{1}-c_{2}}\left[1+o\left(|u|^{-1}\right)\right] .
\end{gathered}
$$

Letting $a=0$ in Example 7, and using (81), we lead to the expression of $\psi_{2}(u)$ as follows:

$$
\begin{aligned}
\psi_{2}(u)= & U\left(\frac{\delta}{2 v}, \frac{1}{2}, \frac{v}{\sigma^{2}}\left(\ln \frac{u}{k}+\frac{\sigma^{2}}{2 v}\right)^{2}\right) \\
& \times\left[U\left(\frac{\delta}{2 v}, \frac{1}{2}, \frac{v}{\sigma^{2}}\left(\ln \frac{b}{k}+\frac{\sigma^{2}}{2 v}\right)^{2}\right)\right]^{-1} .
\end{aligned}
$$

Using (71), we get

$$
\begin{aligned}
\psi_{2}^{\prime}(b)= & -\frac{\delta}{\sigma^{2} b k} \ln \left(\frac{b}{k}+\frac{\sigma^{2}}{2 v}\right) \\
& \times U\left(\frac{\delta}{2 v}+1, \frac{1}{2}+1, \frac{v}{\sigma^{2}}\left(\ln \frac{b}{k}+\frac{\sigma^{2}}{2 v}\right)^{2}\right) \\
& \times\left[U\left(\frac{\delta}{2 v}, \frac{1}{2}, \frac{v}{\sigma^{2}}\left(\ln \frac{b}{k}+\frac{\sigma^{2}}{2 v}\right)^{2}\right)\right]^{-1} .
\end{aligned}
$$

Then, from Theorem 9, we have

$$
\begin{aligned}
V_{r}(u, b)= & -\frac{\sigma^{2} b k}{\delta} U\left(\frac{\delta}{2 v}, \frac{1}{2}, \frac{v}{\sigma^{2}}\left(\ln \frac{u}{k}+\frac{\sigma^{2}}{2 v}\right)^{2}\right) \\
\times & {\left[\ln \left(\frac{b}{k}+\frac{\sigma^{2}}{2 v}\right)\right.} \\
& \left.\times U\left(\frac{\delta}{2 v}+1, \frac{1}{2}+1, \frac{v}{\sigma^{2}}\left(\ln \frac{b}{k}+\frac{\sigma^{2}}{2 v}\right)^{2}\right)\right] .
\end{aligned}
$$

3.2. Threshold Strategy. We consider the company pays dividends according to the threshold dividend strategy; that is, dividends are paid at a constant rate $\alpha$ whenever the modified surplus is above the threshold $b$, and no dividends are paid whenever the modified surplus is below $b$. For recent publications on threshold strategy, see, for example, $[3,16,17]$. We define the modified risk process by

$$
U_{d}(t)=U_{t}-D_{d}(t),
$$

where $D_{d}(t)=\alpha \int_{0}^{t} I\left(U_{d}(s)>b\right) d s$. Let $D_{d}$ denote the present value of all dividends until ruin as follows:

$$
D_{d}=\alpha \int_{0}^{T_{d}} e^{-\delta s} I\left(U_{d}(s)>b\right) d s,
$$

where $T_{d}=\inf \left\{t \geq 0: U_{d}(t)=0\right\}$. We denote by $V_{d}(u, b)$ the expected discounted value of dividend payments; that is,

$$
V_{d}(u, b)=E_{u}\left[D_{d}\right] .
$$

We denote

$$
\begin{gathered}
\tau_{b}^{-}=\inf \left\{t: U_{d}(t) \leq b\right\}, \\
\varphi_{3}(u)=E\left[e^{-\delta \tau_{b}^{-}}\right] .
\end{gathered}
$$

We can mimic the discussion of Theorem 1 to give the differential equation and the boundary conditions satisfied by $\varphi_{3}(u)$.

Lemma 14. The function $\varphi_{3}(u)$ defined by (88) satisfies the following differential equation:

$$
\frac{1}{2} \sigma^{2}(u) \varphi_{3}^{\prime \prime}(u)+(\mu(u)-\alpha) \varphi_{3}^{\prime}(u)=\delta \varphi_{3}(u), \quad u \geq b,
$$

with the boundary conditions $\varphi_{3}(b)=1, \varphi_{3}(\infty)=0$.

We have the following theorem.

Theorem 15. For $u \in[0, b]$, one has

$$
V_{d}(u ; b)=\frac{\alpha}{\delta} \frac{\varphi_{3}^{\prime}(b) \psi_{2}(u)}{\varphi_{3}^{\prime}(b)-\psi_{2}^{\prime}(b)},
$$

and, for $u>b$, one has

$$
V_{d}(u ; b)=\frac{\alpha}{\delta}+\frac{\alpha}{\delta} \frac{\psi_{2}^{\prime}(b) \varphi_{3}(u)}{\varphi_{3}^{\prime}(b)-\psi_{2}^{\prime}(b)} .
$$


Proof. When $u \in[0, b]$, in view of the strong Markov property, we obtain

$$
V_{d}(u ; b)=V_{d}(b ; b) \psi_{2}(u) .
$$

When $u>b$, since $\tau_{b}^{-}$is a stopping time, it follows from the strong Markov property of $U_{d}(u)$ that

$$
\begin{aligned}
V_{d}(u, b)= & E_{u}\left(\int_{0}^{\tau_{b}^{-}} \alpha e^{-\delta t} d t\right) \\
& +E_{u}\left(\int_{\tau_{b}^{-}}^{T_{d}} \alpha e^{-\delta t} I\left(U_{d}(t)>b\right) d t\right) \\
= & \frac{\alpha}{\delta}\left(1-E_{u} e^{-\delta \tau_{b}^{-}}\right) \\
& +E_{u}\left[e^{-\delta \tau_{b}^{-}} E_{U_{\tau_{b}^{-}}}\left(\int_{0}^{T_{d}} e^{-\delta t} d D_{d}(t)\right)\right] \\
= & \frac{\alpha}{\delta}+\left(V_{d}(b, b)-\frac{\alpha}{\delta}\right) \varphi_{3}(u) .
\end{aligned}
$$

Using the continuity of the function $V_{d}^{\prime}(u ; b)$ at $u=b$, we get

$$
V_{d}(b ; b) \psi_{2}^{\prime}(b)=\left(V_{d}(b, b)-\frac{\alpha}{\delta}\right) \varphi_{3}^{\prime}(b) .
$$

So, we get

$$
V_{d}(b ; b)=\frac{\alpha}{\delta} \frac{\varphi_{3}^{\prime}(b)}{\varphi_{3}^{\prime}(b)-\psi_{2}^{\prime}(b)} .
$$

Substituting the above expression into (92) and (93), we can get the results (90) and (91). This completes the proof.

We just consider the square root process.

Example 16. We consider the square root process discussed in Example 11. We first solve the differential equation satisfied by $\varphi_{3}(u)$ as follows

$$
\frac{1}{2} \sigma^{2} u^{2} \varphi_{3}^{\prime \prime}(u)+(v k-v u-\alpha) \varphi_{3}^{\prime}(u)=\delta \varphi_{3}(u), \quad \delta>0 .
$$

We assume that $2\left((v k-\alpha) / \sigma^{2}\right)$ is not an integer, the two linear independent solutions are

$$
\begin{aligned}
& \varphi_{+}(u)=M\left(\frac{\delta}{v}, \frac{2(v k-\alpha)}{\sigma^{2}}, \frac{2 v}{\sigma^{2}} u\right), \\
& \varphi_{-}(u)=U\left(\frac{\delta}{v}, \frac{2(v k-\alpha)}{\sigma^{2}}, \frac{2 v}{\sigma^{2}} u\right) .
\end{aligned}
$$

Using (81), and from the boundary conditions $\varphi_{3}(b)=1$, $\varphi_{3}(\infty)=0$, we get

$$
\begin{aligned}
\varphi_{3}(u)= & {\left[U\left(\frac{\delta}{v}, \frac{2(v k-\alpha)}{\sigma^{2}}, \frac{2 v}{\sigma^{2}} u\right)\right] } \\
& \times\left[U\left(\frac{\delta}{v}, \frac{2(v k-\alpha)}{\sigma^{2}}, \frac{2 v}{\sigma^{2}} b\right)\right]^{-1} .
\end{aligned}
$$

From (71), we have

$$
\begin{aligned}
\varphi_{3}^{\prime}(b)= & -\frac{2 \delta}{\sigma^{2}}\left[U\left(\frac{\delta}{v}+1, \frac{2(v k-\alpha)}{\sigma^{2}}+1, \frac{2 v}{\sigma^{2}} b\right)\right] \\
& \times\left[U\left(\frac{\delta}{v}, \frac{2(v k-\alpha)}{\sigma^{2}}, \frac{2 v}{\sigma^{2}} b\right)\right]^{-1} .
\end{aligned}
$$

Furthermore, $\psi_{2}(u)$ and $\psi_{2}^{\prime}(b)$ have been given in Example 11. From Theorem 15, we obtain

$$
\begin{aligned}
V_{d}(u, b)=\frac{\alpha}{\delta} U\left(\frac{\delta}{v}+1, \frac{2(v k-\alpha)}{\sigma^{2}}+1, \frac{2 v}{\sigma^{2}} b\right) \\
\times\left[U\left(\frac{\delta}{v}, \frac{2 v}{\sigma^{2}} k, 0\right) M\left(\frac{\delta}{v}, \frac{2 v}{\sigma^{2}} k, \frac{2 v}{\sigma^{2}} u\right)\right. \\
\left.\quad-U\left(\frac{\delta}{v}, \frac{2 v}{\sigma^{2}} k, \frac{2 v}{\sigma^{2}} u\right)\right] \\
\times\left\{C U\left(\frac{\delta}{v}+1, \frac{2(v k-\alpha)}{\sigma^{2}}+1, \frac{2 v}{\sigma^{2}} b\right)\right. \\
+U\left(\frac{\delta}{v}, \frac{2(v k-\alpha)}{\sigma^{2}}, \frac{2 v}{\sigma^{2}} b\right) \\
\times\left[\frac{\sigma^{2}}{2 v k} U\left(\frac{\delta}{v}, \frac{2 v}{\sigma^{2}} k, 0\right)\right. \\
\quad \times M\left(\frac{\delta}{v}+1, \frac{2 v}{\sigma^{2}} k+1, \frac{2 v}{\sigma^{2}} b\right) \\
\left.\left.+U\left(\frac{\delta}{v}+1, \frac{2 v}{\sigma^{2}} k+1, \frac{2 v}{\sigma^{2}} b\right)\right]\right\},
\end{aligned}
$$

and for $u>b$, we get

$$
\begin{aligned}
& V_{d}(u, b)= \frac{\alpha}{\delta}-\frac{\alpha}{\delta} U\left(\frac{\delta}{v}, \frac{2(v k-\alpha)}{\sigma^{2}}, \frac{2 v}{\sigma^{2}} u\right) \\
& \times\left[\frac{\sigma^{2}}{2 v k} U\left(\frac{\delta}{v}, \frac{2 v}{\sigma^{2}} k, 0\right)\right. \\
& \times M\left(\frac{\delta}{v}+1, \frac{2 v}{\sigma^{2}} k+1, \frac{2 v}{\sigma^{2}} b\right) \\
&\left.\quad+U\left(\frac{\delta}{v}+1, \frac{2 v}{\sigma^{2}} k+1, \frac{2 v}{\sigma^{2}} b\right)\right] \\
& \times\left\{C U\left(\frac{\delta}{v}+1, \frac{2(v k-\alpha)}{\sigma^{2}}+1, \frac{2 v}{\sigma^{2}} b\right)\right. \\
& \quad+U\left(\frac{\delta}{v}, \frac{2(v k-\alpha)}{\sigma^{2}}, \frac{2 v}{\sigma^{2}} b\right)
\end{aligned}
$$




$$
\begin{aligned}
& \times\left[\frac{\sigma^{2}}{2 v k} U\left(\frac{\delta}{v}, \frac{2 v}{\sigma^{2}} k, 0\right)\right. \\
& \times M\left(\frac{\delta}{v}+1, \frac{2 v}{\sigma^{2}} k+1, \frac{2 v}{\sigma^{2}} b\right) \\
& \left.\left.+U\left(\frac{\delta}{v}+1, \frac{2 v}{\sigma^{2}} k+1, \frac{2 v}{\sigma^{2}} b\right)\right]\right\}^{-1},
\end{aligned}
$$

where $C$ is defined in Example 11.

\section{Conflict of Interests}

The authors declare that there is no conflict of interests regarding the publication of this paper.

\section{Acknowledgments}

The authors are grateful to the anonymous referee's careful reading and detailed helpful comments and constructive suggestions, which have led to a significant improvement of the paper. The research was supported by the National Natural Science Foundation of China (no. 11171179), the Research Fund for the Doctoral Program of Higher Education of China (no. 20133705110002), and the Program for Scientific Research Innovation Team in Colleges and Universities of Shandong Province.

\section{References}

[1] S. Asmussen and M. Taksar, "Controlled diffusion models for optimal dividend pay-out," Insurance, vol. 20, no. 1, pp. 1-15, 1997.

[2] J. Cai, H. U. Gerber, and H. Yang, "Optimal dividends in an Ornstein-Uhlenbeck type model with credit and debit interest," North American Actuarial Journal, vol. 10, no. 2, pp. 94-119, 2006.

[3] Y. Fang and R. Wu, "Optimal dividends in the Brownian motion risk model with interest," Journal of Computational and Applied Mathematics, vol. 229, no. 1, pp. 145-151, 2009.

[4] C. C. Yin and H. Q. Wang, "The first passage time and the dividend value function for one-dimension diffusion processes between two reflecting barriers," International Journal of Stochastic Analysis, vol. 2012, Article ID 971212, 15 pages, 2012.

[5] C. C. Yin, Y. Shen, and Y. Z. Wen, "Exit problems for jump processes with applications to dividend problems," Journal of Computational and Applied Mathematics, vol. 245, pp. 30-52, 2013.

[6] S. Ditlevsen, "A result on the first-passage time of an OrnsteinUhlenbeck process," Statistics and Probability Letters, vol. 77, no. 18, pp. 1744-1749, 2007.

[7] J. C. Cox, J. E. Ingersoll Jr., and S. A. Ross, "A theory of the term structure of interest rates," Econometrica, vol. 53, no. 2, pp. 385407, 1985.

[8] Y. Madec and C. Japhet, "First passage time problem for a drifted Ornstein-Uhlenbeck process," Mathematical Biosciences, vol. 189, no. 2, pp. 131-140, 2004.
[9] L. Wang and K. Pötzelberger, "Crossing probabilities for diffusion processes with piecewise continuous boundaries," Methodology and Computing in Applied Probability, vol. 9, no. 1, pp. 2140, 2007.

[10] H. U. Gerber and E. S. W. Shiu, "On the time value of ruin," North American Actuarial Journal, vol. 2, no. 1, pp. 48-78, 1998.

[11] H. U. Gerber and E. S. W. Shiu, "Optimal dividends: analysis with Brownian motion," North American Actuarial Journal, vol. 8, no. 1, pp. 1-20, 2004.

[12] C. W. Wang, C. C. Yin, and E. Q. Li, "On the classical risk model with credit and debit interests under absolute ruin," Statistics and Probability Letters, vol. 80, no. 5-6, pp. 427-436, 2010.

[13] K. C. Yuen and C. Yin, "On optimality of the barrier strategy for a general Lévy risk process," Mathematical and Computer Modelling, vol. 53, no. 9-10, pp. 1700-1707, 2011.

[14] S. J. Zhang and J. M. Jin, Computation of Special Functions, Nanjing University Press, Nanjing, China, 2011.

[15] Abramowitz and M. Stegun, Handbook of Mathematical Functions: With Formulas, Graphs, and Mathematical Tables, United States Department of Commerce, US Government Printing Office, Washington, DC, USA, 1972.

[16] Y. Chi and X. S. Lin, "On the threshold dividend strategy for a generalized jump-diffusion risk model," Insurance, vol. 48, no. 3, pp. 326-337, 2011.

[17] A. C. Y. Ng, "On a dual model with a dividend threshold," Insurance, vol. 44, no. 2, pp. 315-324, 2009. 


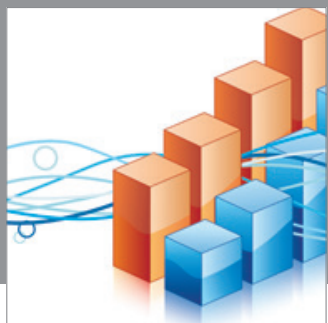

Advances in

Operations Research

mansans

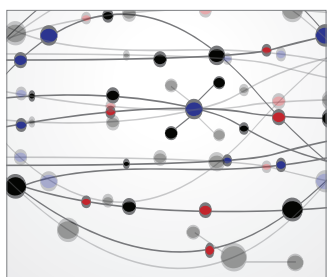

The Scientific World Journal
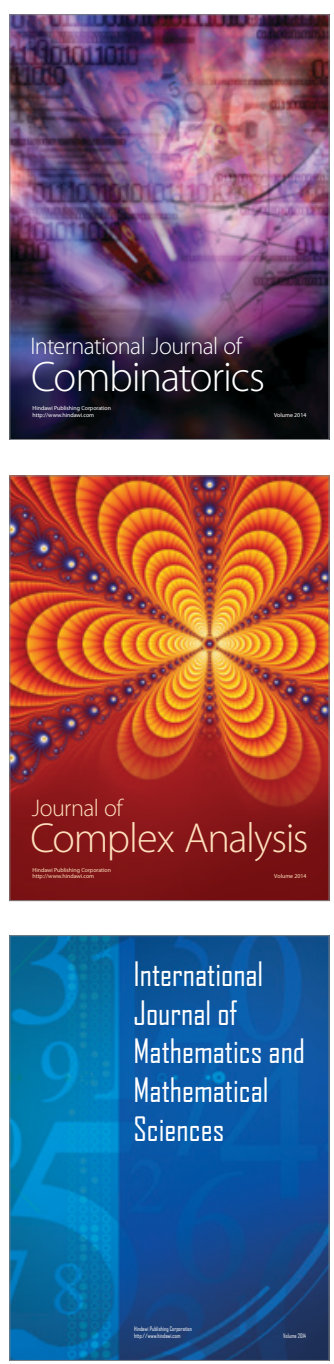
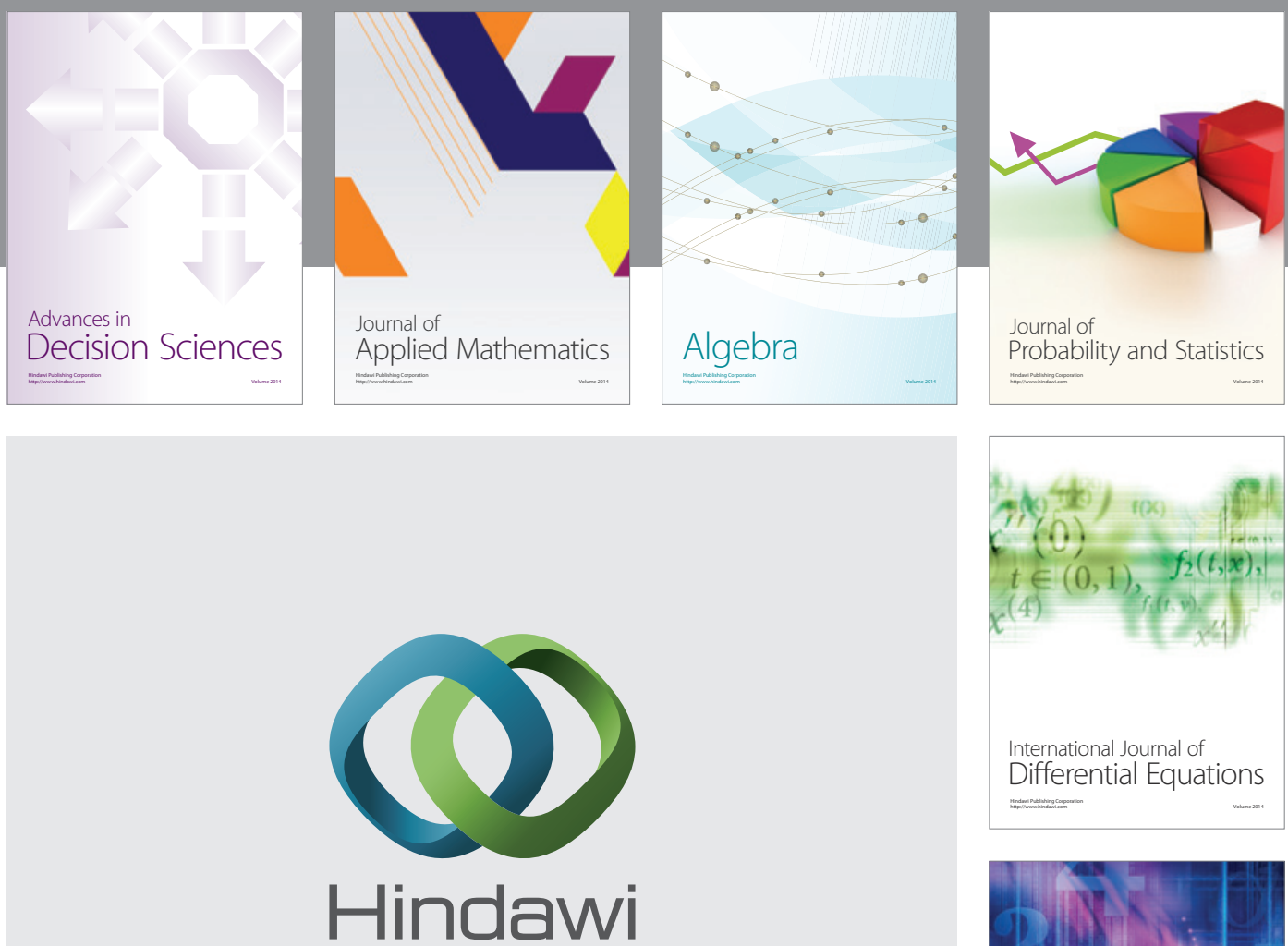

Submit your manuscripts at http://www.hindawi.com
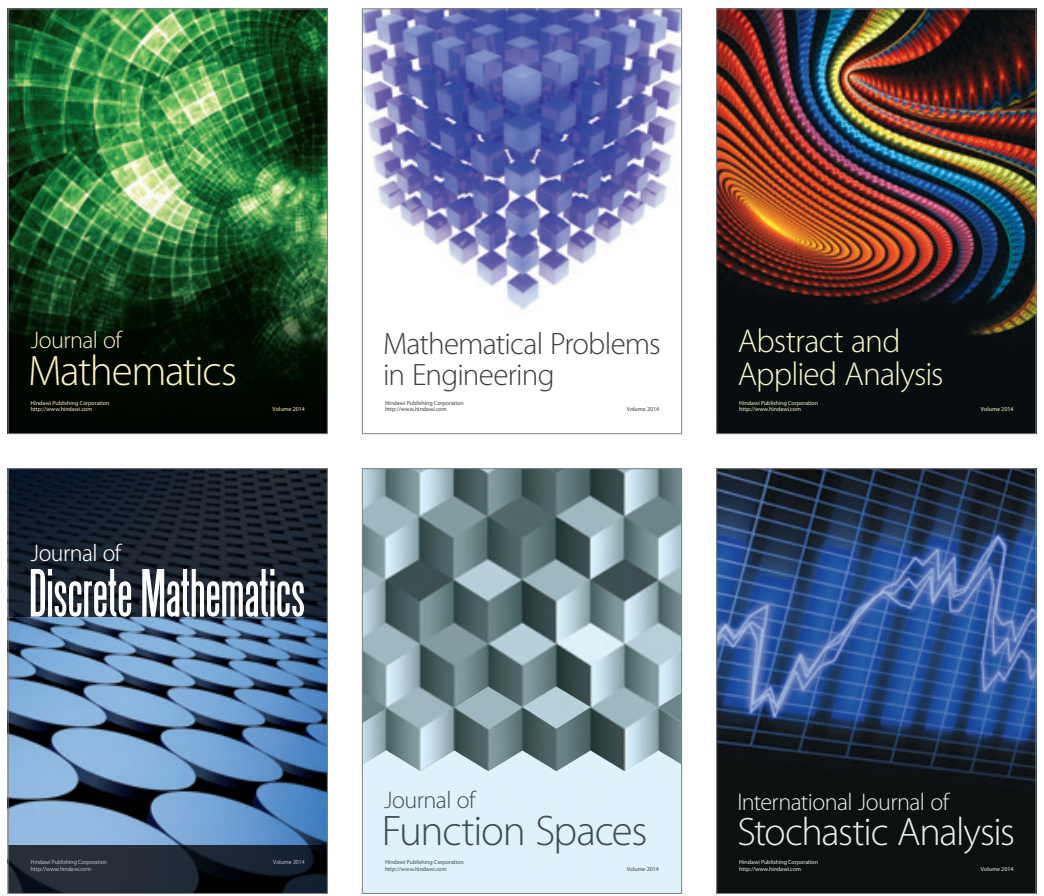

Journal of

Function Spaces

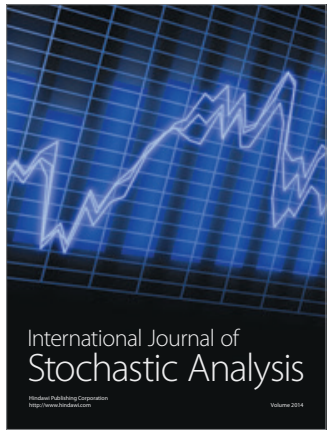

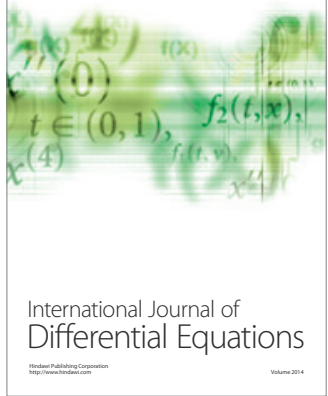
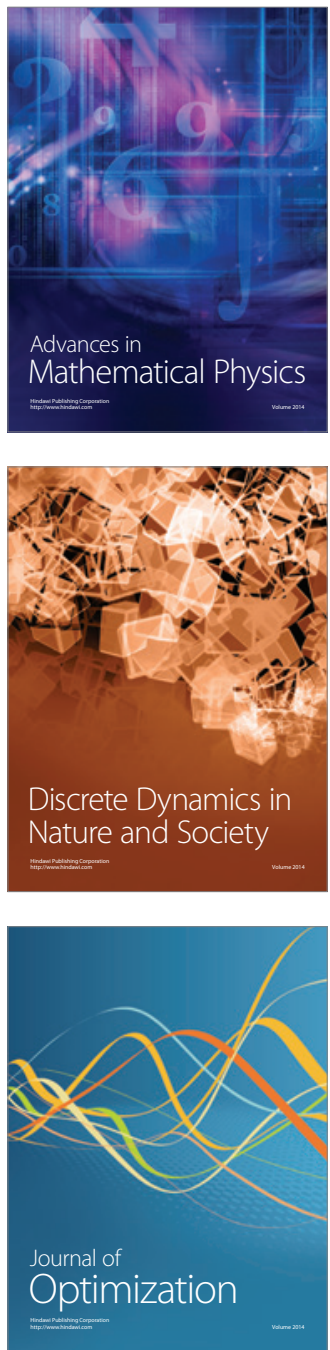